\title{
Expression of PSMA in tumor neovasculature of high grade sarcomas including synovial sarcoma, rhabdomyosarcoma, undifferentiated sarcoma and MPNST
}

\author{
Birthe Heitkötter ${ }^{1}$, Marcel Trautmann', Inga Grünewald ${ }^{1}$, Martin Bögemann ${ }^{2}$, \\ Kambiz Rahbar ${ }^{3}$, Heidrun Gevensleben ${ }^{4}$, Eva Wardelmann ${ }^{1}$, Wolfgang Hartmann ${ }^{1}$, \\ Konrad Steinestel ${ }^{1}$ and Sebastian Huss ${ }^{1}$ \\ ${ }^{1}$ Gerhard Domagk Institute of Pathology, University Hospital Münster, University of Münster, Germany \\ 2 Department of Urology, University Hospital Münster, University of Münster, Germany \\ ${ }^{3}$ Department of Nuclear Medicine, University Hospital Münster, University of Münster, Germany \\ ${ }^{4}$ Institute of Pathology, University Hospital Bonn, University of Bonn, Germany \\ Correspondence to: Birthe Heitkötter, email: Birthe.Heitkoetter@ukmuenster.de
}

Keywords: PSMA, sarcoma, neovasculature, soft tissue tumor, therapy

Received: August 20,2016 Accepted: November 30,2016 Published: December 16, 2016

\section{ABSTRACT}

Aims: PSMA (prostate specific membrane antigen) is physiologically expressed in normal prostate tissue. It is overexpressed in prostate cancer cells and has been suggested as a target for antibody-based radioligand therapy. As PSMA expression so far has not been systematically analyzed in soft tissue tumors, the current study aims at investigating a large cohort of different subtypes.

Methods and Results: Immunohistochemistry was used to detect PSMA expression in 779 samples of soft tissue tumors and Ewing sarcoma as a primary bone malignancy. CD34 coexpression was employed to study PSMA expression in the neovasculature. PSMA expression was found in the tumor-associated neovasculature of 151/779 soft tissue/bone tumors $(19.38 \%)$ and was more frequent in malignant tumors compared to tumors with intermediate or benign biological potential $(p=0.078)$. Strong neovascular PSMA expression was predominantly observed in subsets of different sarcomas including 3/20 rhabdomyosarcomas (15\%), 4/21 malignant peripheral nerve sheath tumors $(19.05 \%), 6 / 16$ synovial sarcomas $(35.29 \%)$ and $6 / 33$ undifferentiated pleomorphic sarcomas (18.18\%).

Conclusion: We conclude that PSMA is expressed in the neovasculature of a subset of soft tissue tumors to a variable extent. Our observation of strong PSMA expression predominantly occurring in sarcomas might provide a rationale to evaluate PSMA-targeted radioligand therapy in these entities.

\section{INTRODUCTION}

PSMA (prostate specific membrane antigen) is a $100 \mathrm{kDa}$ type II transmembrane glycoprotein carrying intra- and extracellular protein domains. Functionally, it exerts a folate hydrolase and neurocarboxypeptidase activity $[1,2]$. It was originally found to be physiologically expressed by prostate cells and other tissues (e.g. small intestine, renal tubules or salivary glands) [3, 4]. The discovery of its strong upregulation in prostate cancer cells, however, yielded PSMA-based imaging for the detection of metastatic disease in advanced prostate cancer. PSMA expression was further shown to correlate with prostate cancer grade and was substantiated as an independent predictive factor for tumor recurrence [5]. Moreover, PSMA-based radioligand therapy has been established as a therapeutic regimen in metastasized prostate cancer [2, 6-12].

Surprisingly, PMSA was found to be expressed in the endothelium of tumor-associated neovasculature in some solid malignancies, possibly due to the effect of tumor-associated angiogenic factors [13-17]. Among 
Table 1: TMA composition with the numbers of cases included $(n=779)$

\begin{tabular}{|c|c|}
\hline Subgroup & Entity \\
\hline Adipocytic & $\begin{array}{l}\text { Lipoma }(2) \\
\text { Liposarcoma (152) } \\
\text { • Well differentiated 67) } \\
\text { - Dedifferentiated (75) } \\
\text { - Pleomorphic (10) } \\
\text { Myxoid liposarcoma (30) }\end{array}$ \\
\hline Skeletal-muscle tumor* & $\begin{aligned} & \text { Rhabdomyosarcoma }(20) \\
& \text { - } \text { embryonal (7) } \\
& \text { - } \text { alveolar }(8) \\
& \text { - } \text { pleomorphic }(5)\end{aligned}$ \\
\hline Smooth-muscle tumor & $\begin{array}{l}\text { Leiomyoma (6) } \\
\text { Leiomyosarcoma (66) }\end{array}$ \\
\hline Vascular tumor & $\begin{array}{l}\text { Haemangioma (6) } \\
\text { Angiosarcoma of soft tissue (29) }\end{array}$ \\
\hline Nerve sheath tumor & $\begin{array}{l}\text { Schwannoma (14) } \\
\text { Neurofibroma (2) } \\
\text { Ganglioneuroma (2) } \\
\text { Malignant peripheral nerve sheath tumor (21) }\end{array}$ \\
\hline Fibroblastic/myofibroblastic tumors & $\begin{array}{l}\text { Inflammatory myofibroblastic tumor (2) } \\
\text { Extrapleural solitary fibrous tumor (35) } \\
\text { Desmoid-type fibromatosis (44) } \\
\text { Myxofibrosarcoma (6) }\end{array}$ \\
\hline Gastrointestinal stromal tumor & Gastrointestinal stromal tumor (183) \\
\hline Tumor of uncertain differentiation & Synovial Sarcoma (16) \\
\hline Undifferentiated sarcomas & $\begin{array}{l}\text { Undifferentiated pleomorphic sarcoma (33) } \\
\text { Endometrial stromal sarcoma (4) }\end{array}$ \\
\hline Primary bone tumors & Ewing-Sarcoma (106) \\
\hline
\end{tabular}

others, PSMA expression in endothelial neovasculature was shown for clear cell renal carcinomas, transitional cell carcinomas of the urinary bladder, colonic adenocarcinomas, glioblastoma multiforme, lung cancers, malignant melanomas, schwannomas and osteosarcomas [13-15, 18, 19]. In osteosarcomas, PSMA expression in tumor-associated neovasculature furthermore correlated with tumor size, pulmonary metastasis and unfavorable clinical course [19]. The role of PSMA in tumor angiogenesis is part of an autoregulatory loop involving $\beta 1$-integrin and p21-activated kinase 1 (PAK1). In this loop, active PSMA facilitates endothelial cell invasion through the extracellular matrix by interacting with the cytoskeleton via integrin signaling and actin-binding protein Filamin A $[8,20]$. These findings have been supported by Gordon et al. who reported that PSMA expression is also increased in nonneoplastic, regenerative, and reparative neovasculature (scars, granulation tissue and proliferative endometrium). The authors proposed that the folate hydrolase activity of PSMA enhances the local availability of folic acid, facilitating angiogenesis by increasing the levels of proangiogenic nitric oxide through regeneration of endothelial nitric oxide synthase [8].

Since PSMA expression is not exclusively limited to prostate cancer but also found in the neovasculature of solid epithelial cancers, these results might imply the potential option of new radioligand-based or antiangiogenic therapeutics. As soft tissue tumors have not been systematically analyzed for PSMA expression, the current study aims at investigating a large cohort of different entities, including aggressive subgroups of tumors.

\section{RESULTS}

\section{PSMA expression in tumor neovasculature}

PSMA expression in the tumor-associated neovasculature was found to be positive in 151 of 779 soft tissue/bone tumors (19.38\%): 2/67 well differentiated liposarcomas $\quad(2.99 \%), \quad 17 / 75 \quad$ dedifferentiated liposarcomas $(22.67 \%), 5 / 10$ pleomorphic liposarcomas $(50 \%), 1 / 30$ myxoid liposarcomas $(3.34 \%), 0 / 2$ lipomas (0\%), 1/7 embryonal rhabdomyosarcomas (14.29\%), 3/5 pleomorphic rhabdomyosarcomas (60\%), 1/8 alveolar rhabdomyosarcomas $(12.5 \%), 0 / 6$ leiomyomas $(0 \%)$, 21/66 leiomyosarcomas (31.81\%), 2/6 haemangiomas (33.34\%), 3/29 angiosarcomas of soft tissue (10.34\%), $3 / 14$ schwannomas $(21.43 \%), 7 / 21$ malignant peripheral nerve sheath tumors $(33.34 \%), 0 / 2$ neurofibromas $(0 \%)$, 
Table 2: Numbers of tumors with strong PSMA expression in the neovasculature (PSMA labelling index $=2$; total $n=43$ )

\begin{tabular}{|c|c|c|}
\hline Subgroup & $\mathbf{n}$ & Entity \\
\hline Adipocytic & 2 & $\begin{array}{l}\text { 0/2 Lipoma } \\
\text { 0/67 Well differentiated liposarcoma } \\
\text { 0/75 Dedifferentiated liposarcoma } \\
\text { 2/20 Pleomorphic liposarcoma } \\
\text { 0/30 Myxoid liposarcoma }\end{array}$ \\
\hline Skeletal-muscle & 3 & $\begin{array}{l}\text { 0/7 Embryonal rhabdomyosarcoma } \\
\text { 1/8 Alveolar rhabdomyosarcoma* } \\
\text { 2/5 Pleomorphic rhabdomyosarcoma }\end{array}$ \\
\hline Smooth-muscle & 7 & $\begin{array}{l}\text { 0/6 Leiomyoma } \\
\text { 7/66 Leiomyosarcoma }\end{array}$ \\
\hline Vascular tumor & 3 & $\begin{array}{l}\text { 0/6 Haemangioma } \\
\mathbf{3 / 2 9} \text { Angiosarcoma of soft tissue }\end{array}$ \\
\hline Nerve sheath tumor & 5 & $\begin{array}{l}\text { 1/14 Schwannoma } \\
\text { 0/2 Neurofibroma } \\
\text { 0/2 Ganglioneuroma } \\
\text { 4/21 Malignant peripheral nerve sheath tumor }\end{array}$ \\
\hline $\begin{array}{l}\text { Fibroblastic/myofibroblastic } \\
\text { tumors }\end{array}$ & 3 & $\begin{array}{l}\text { 0/2 Inflammatory myofibroblastic tumor } \\
\text { 1/35 Extrapleural solitary fibrous tumor } \\
\text { 2/44 Desmoid-type fibromatosis } \\
\text { 0/6 Myxofibrosarcoma }\end{array}$ \\
\hline Gastrointestinal stromal tumor & 2 & 2/183 Gastrointestinal stromal tumor \\
\hline Tumor of uncertain differentiation & 6 & 6/16 Synovial sarcoma \\
\hline Undifferentiated sarcomas & 6 & $\begin{array}{l}\text { 6/33 Undifferentiated pleomorphic sarcoma } \\
\text { 0/4 Endometrial stromal sarcoma }\end{array}$ \\
\hline Primary bone tumors & 6 & 6/106 Ewing-Sarcoma \\
\hline
\end{tabular}

Entities with more than one tumor and strong PSMA expression in at least $10 \%$ of the tumor's neovasculature are indicated in bold.

* In one rhabdomyosarcoma a strong tumor cell expression was observed.

This entity was therefore analyzed in a validation study containing further 16 rhabdomyosarcomas.

No additional case showing a tumor cell expression was found.

0/2 ganglioneuromas $(0 \%), 1 / 6$ myxofibrosarcomas (16.67\%), 12/35 extrapleural solitary fibrous tumors $(34.29 \%), 0 / 2$ inflammatory myofibroblastic tumors (0\%), 10/44 desmoid-type fibromatosis (22.73\%), 14/106 Ewing sarcomas (13.21\%), 23/183 gastrointestinal stromal tumors $(12.57 \%), 9 / 16$ synovial sarcomas $(56.25 \%)$, $15 / 33$ undifferentiated pleomorphic sarcomas and 1/4 endometrial stromal sarcomas $(25 \%)$. Of these tumors, the majority $(108 / 779 ; 13.86 \%)$ showed low expression levels while $5.52 \%$ percent (43/779) showed strong PSMA expression (see table 2 and Figure 2). Detailed data on staining intensity and distribution patterns for each evaluated tumor is shown in Supplementary Ttable 1.

We then performed a histogram analysis linking the biological potential of different tumors with the neovascular PSMA labelling index (Figure 2). The overall PSMA expression (low and high expression, labelling index 1 and 2) was found in the neovasculature of 122/599 (20.37\%) malignant tumors, whereas PSMA expression in the neovasculature of tumors with intermediate biological potential $(24 / 148(16.22 \%)$ and benign tumors $(5 / 32$ $(15.63 \%))$ tended to be lower $(p=0.237$, Fisher's exact test, malignant $v s$. intermediate/benign tumors).
Analyzing only tumors with strong neovascular PSMA expression (labelling index 2), malignant tumors with a high PSMA expression were more frequent (39/599; $6.51 \%$ ) compared to tumors of intermediate biological potential $(3 / 148 ; 2.03 \%)$ and benign tumors $(1 / 32 ; 3.13 \%)$ ( $p=0.078$, Fisher's exact test, malignant $v s$. intermediate/ benign tumors).

According to the FNCLCC grading of sarcomas, some tumors are designated as high grade sarcomas (grade 3) by diagnosis, e.g. Ewing sarcomas, rhabdomyosarcomas (except spindle cell and botryoid variants), angiosarcomas and pleomorphic liposarcomas. Focusing only on the PSMA expression in the neovasculature of these high grade sarcomas, we noted that the neovasculature of $2 / 20$ pleomorphic liposarcomas (10\%), 3/29 angiosarcomas of the soft tissue $(10.34 \%), 1 / 8$ alveolar rhabdomyosarcomas $(12.50 \%)$ and $2 / 5$ pleomorphic rhabdomyosarcomas (40\%) showed a strong expression (labelling index $=2$ ) in more than $10 \%$ of the investigated cases, whereas only $6 / 106$ $(5.66 \%)$ of the investigated Ewing sarcomas presented a strong expression (labelling index $=2$ ) in the tumorassociated neovasculature.

Regarding all represented entities, the highest 
percentage of a strong PSMA expression (labelling index $=2$ ) could be found in the neovasculature of pleomorphic rhabdomyosarcomas $(40 \%)$, followed by synovial sarcomas $(37.50 \%)$, malignant peripheral nerve sheath tumors $(19.05 \%)$, undifferentiated pleomorphic sarcomas $(18.19 \%)$, alveolar rhabdomyosarcomas (12.50\%), leiomyosarcomas $(10.61 \%)$, angiosarcomas of the soft tissue (10.34\%) and pleomorphic liposarcomas (10\%).

\section{PSMA expression in tumor cells}

We observed a very strong cellular PSMA expression in one case of alveolar rhabdomyosarcoma (inset Figure 1). This alveolar rhabdomyosarcoma was found in the right upper lung lobe of a 83 year old male patient. Before undergoing surgery, the lesion was thought to be non-small cell lung cancer. The patient had no history of prostate cancer and his PSA-levels were normal. Immunohistological staining showed no expression of

cytokeratins (AE1/3, CK7, CK18) or S-100 and a weak expression of CD56. There was a strong expression of vimentin and desmin as well as a nuclear positivity for Myo-D1 and Myogenin. Staining against caldesmon and smooth muscle actin was negative. A translocation involving the FOXO1 gene on chromosome $13 \mathrm{q} 14$ could be excluded by fluorescence in situ hybridization (FISH). The tumor was finally signed out as translocation-negative alveolar rhabdomyosarcoma (solid variant).

\section{Representativeness of tissue microarrays}

To rule out a selection bias by IHC analysis of tissue microarrays (TMAs), we performed additional stainings of whole slides from 12 tumors of benign biological potential (lipoma and hemangioma), 13 tumors with intermediate biological potential (desmoid type fibromatosis) and 12 high grade sarcomas (synovial sarcoma and MPNST) (Supplementary Table 2). PSMA staining of tumor-
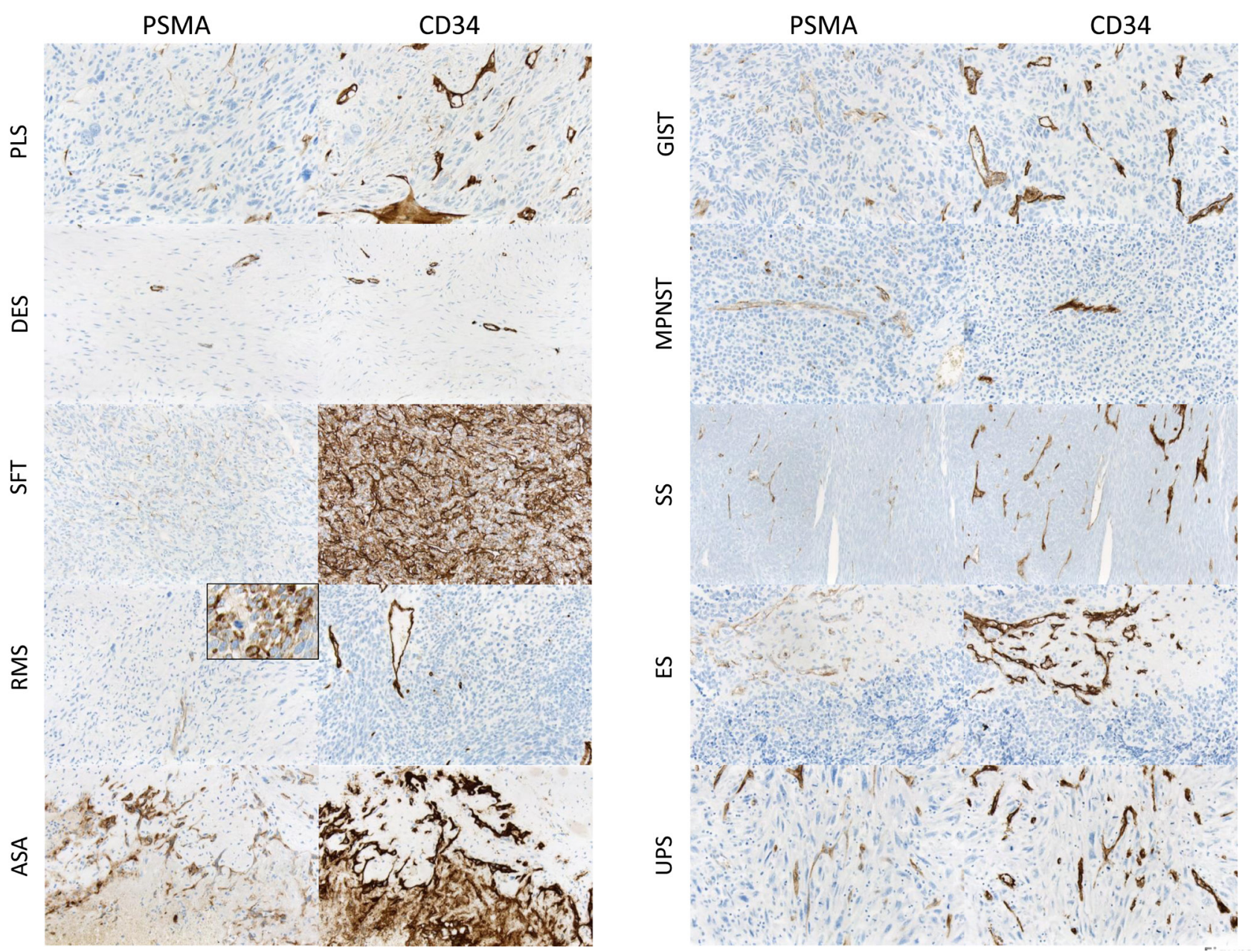

Figure 1: PSMA expression in the neovasculature of sarcomas. Neovascular PSMA expression in different sarcoma subtypes. Vasculature was identified by means of CD34 coexpression. (PLS, pleomorphic liposarcoma; DES, desmoid-type fibromatosis; SFT, solitary fibrous tumor; RMS, rhabdomyosarcoma; ASA, angiosarcoma; GIST, gastrointestinal stromal tumor; MPNST, malignant peripheral nerve sheath tumor; SS, synovial sarcoma; ES, Ewing sarcoma; UPS, undifferentiated pleomorphic sarcoma). In one case of RMS a significant PSMA expression of tumor cells (inset) was observed. 
associated neovasculature could be observed in two additional whole slide cases $(5.4 \%)$ that had previously been classified as "negative" based on the TMA staining. There were no differences with regard to the rate of PSMA staining of tumor cells.

\section{DISCUSSION}

Apart from its known strong cellular expression in prostate cancer, PSMA is expressed in the tumor neovasculature of different solid epithelial cancer subtypes. This finding prompted us to systematically analyze a large cohort of different soft tissue tumors for neovascular as well as intratumoral PSMA expression. We found strong neovascular PSMA expression in a subset of different malignant soft tissue tumors including pleomorphic liposarcoma, rhabdomyosarcoma, leiomyosarcoma, angiosarcoma, MPNST, synovial sarcoma and undifferentiated sarcoma. In addition, one case of rhabdomyosarcoma showed cytoplasmic PSMA expression. Overall, neovascular PSMA expression was more frequent in malignant tumors than in tumors with intermediate or benign biological potential. However, in schwannomas, we found neovascular PSMA-expression in three of 14 cases $(21.42 \%)$. A similar finding was reported by Wang et al. who found an expression of PSMA in the neovasculature of schwannomas in nine of 11 cases [18].

Today, we distinguish over 50 histological types of soft tissue sarcomas with distinct histological appearance and clinical behavior [21]. Especially in advanced disease, clinical management of soft tissue sarcomas requires a multidisciplinary approach [22]. Representing the most reproducible and reliable prognostic factor for survival, complete (R0) resection remains the cornerstone of treatment for resectable soft tissue sarcomas. Therefore, the only curative loco-regional approach as well as the usual first-line treatment of these tumors is wide margin surgery. Radiotherapy represents a further therapeutic tool shown to significantly improve outcome of patients with soft tumors. In contrast, the therapeutic role of conventional chemotherapy in advanced soft tissue sarcomas is limited. Anthracycline-based chemotherapy,

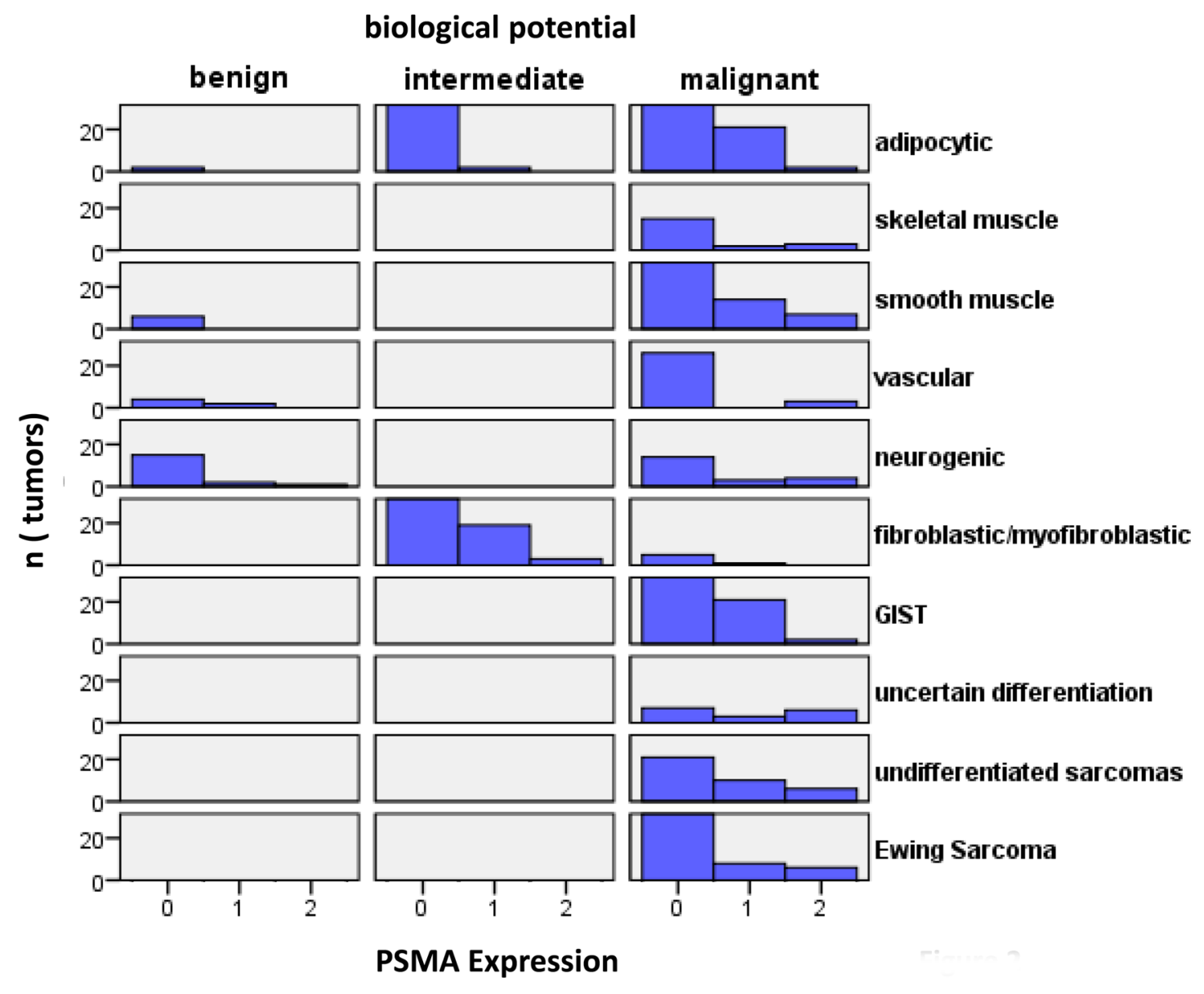

Figure 2: Histograms of tumors according to their biological potential and PSMA labelling index. Histograms of tumors according to their biological potential and PSMA labeling index. 
which is the first-line treatment for most advanced soft tissue sarcomas [22], has a response rate of only $\sim 26$ $\%$ [23]. Response rates for Ifosfamide (with or without doxorubicin), another first-line treatment, are as low as $\sim 25 \%$ [24]. After failure of first-line therapy, gemcitabine/ docetaxel offers another therapeutic possibility but treatment options beyond this approach are rare [22]. In metastatic disease, a conventional-dose, singleagent chemotherapy is executed [22]. Due to the lack of reproducible impact on survival (in part because trials include only small numbers of patients with heterogeneous groups of histological/molecular subtypes) the effectiveness of adjuvant chemotherapy after resection of high-grade soft tissue sarcomas remains controversial. In a few trials, a lower risk for local recurrence was observed among patients receiving adjuvant chemotherapy, however, without any significant gain in overall survival [25].

In the light of these findings, there is an urgent need for new therapeutic options, especially for advanced soft tissue sarcomas. Since PSMA is expressed in the neovasculature of different solid epithelial cancers and represents a possible target for radioligand-based or antiangiogenic therapeutic strategies, we aimed at investigating PSMA expression in soft tissue and bone tumors as well as in the tumor-associated neovasculature. Our idea was supported by two recent studies evaluating PSMA-targeted therapies in different solid cancer subtypes.

In a phase I trial Milowsky et al. demonstrated that tumor-associated neovasculature in multiple advanced metastatic solid cancers could be targeted with an Indium-111- labeled antibody (J591) binding the extracellular domain of PSMA. Imaging with a chest $\mathrm{x}$-ray, computed tomography scan or magnetic resonance imaging (MRI) by tumor type was as follows: $7 / 10$ kidney (70\%), 4/4 colorectal (100\%), 3/3 lung (100\%), 1/1 bladder (100\%), 3/3 pancreas (100\%) and 1/1 melanoma $(100 \%)$. However, no objective tumor regression could be observed whereas toxicity was acceptable [26].

Another phase I study using the PSMA-targeted docetaxel-containing nanoparticle BIND-014 in patients with advanced solid tumors was recently performed [27]. BIND-014 showed noteworthy activity in multiple tumor types leading to complete response in one case, a 46-yearold female with cervical cancer metastatic to lymph nodes. Radiographically confirmed partial responses could be detected in an ampullary adenocarcinoma metastatic to the liver, in a $K R A S$-mutant lung adenocarcinoma and in cases of breast and gastroesophageal cancer. The authors also reported that several BIND-014-sensitive tumors displayed a moderate to high expression of PSMA, while others did not. The authors concluded that high PSMA expression increases uptake of BIND-014 in certain tumors, pointing towards a potential utility of PSMA expression as a predictive biomarker for responsiveness to BIND-014.

For patients with prostate cancer, the PSMA-targeted radionuclide therapy has been shown to be a therapeutic and diagnostic option [6, 28]. PSMA-617 seems to be the most promising ligand for diagnostics and therapy of prostate cancer metastases and recurrences. Developed by the German Cancer Research Centre (DKFZ) in Heidelberg, PSMA-617 is an important theranostic compound [29]. Several studies using Lutetium-177 labeled PSMA-617 in patients with metastatic castrate resistant prostate cancer have shown respectable response values and acceptable toxicity profile [7, 28, 30]. Future studies using Lutetium-177-PSMA-617 complementary to established therapeutics or randomized placebo controlled trials have to evaluate the therapeutic effect of this new agent in response and survival of patients with metastatic prostate cancer.

In the light of these different studies, our immunohistochemical finding of strong neovascular PSMA expression in a subset of different malignant soft tissue sarcomas seems promising, since it points towards a possible use of PSMA-targeted radioligand or antibodybased antiangiogenic therapy for these aggressive tumors. The actual rate of sarcomas with PSMAexpressing neovasculature might even be slightly higher, since we found a low rate of false-negative cases when immunohistochemistry is performed on TMAs instead of whole slides. The results of our study support first in human imaging studies to evaluate tracer uptake and retention in the most promising sarcoma entities. In the event of high PSMA uptake and long retention in tumor tissue this might then point towards further studies for the evaluation of radioligand therapy using radiolabeled PSMA ligands.

\section{MATERIALS AND METHODS}

\section{Tissue microarrays (TMAs)}

We used 23 different TMAs containing at least two representative cores (core diameter $1 \mathrm{~mm}$ ) of different soft tissue tumors. Selected tumor areas were confirmed by two experienced pathologists before and after TMA construction. As one rhabdomyosarcoma case displayed a strong PSMA tumor cell expression, we decided to select 16 additional rhabdomyosarcomas cases from our archival files. For these cases, whole slides were used to study the expression of PSMA. In total, 779 different tumors were investigated (Table 1). To address the question whether the rate of PSMA positive samples is systematically underestimated by the TMA approach we analyzed a small subset of tumors $(n=37)$ both on TMA and whole slide sections (Supplementary Table 2). The study was approved by the local ethics committee (Az. 2016-091-f-S). 


\section{Immunohistochemistry}

Immunhistochemistry (IHC) was performed on $4-\mu \mathrm{m}$-thick paraffin sections using the peroxidaseconjugated avidin-biotin method. Antibodies included a monoclonal mouse anti-PSMA antibody (clone 3E6, Ventana, Germany, 1:50 dilution) and a monoclonal antiCD34 antibody (clone QBEnd10, Ventana, Germany, ready to use concentration of $0.8 \mu \mathrm{g} / \mathrm{ml})$. In brief, sections were deparaffinized in xylene and rehydrated through graded ethanol at room temperature. Incubation with the primary antibodies was performed for 30 minutes at room temperature. After washing, the sections were incubated with biotinylated secondary antibodies. Immunoreactions were visualized using a 3-amino-9-ethylcarbazole as a substrate (Ventana Optiview DAB IHC detection KIT, Ref: 760-700, Germany). Prostate carcinoma tissue sections served as a positive control. Specificity of the PSMA antibody was demonstrated by western immunoblotting of 22RV1 prostate cancer whole cell lysate (data not shown).

\section{Assessment of PSMA expression}

PSMA expression was evaluated by two experienced pathologists (BH and $\mathrm{SH}$ ) on immunostained TMA slides. Tumor cells and associated neovascular endothelium were analyzed separately and the identity of vascular structures was confirmed by CD34 coexpression, a common marker for endothelial cells [31-33].

Any reactivity, either in tumor cells or neoplastic vessels, was considered positive. Staining intensity was scored semiquantitatively as negative (0), weak $(1=$ barely perceptible staining at high power $(400 x)$ magnification), moderate $(2=$ readily apparent at low power (40x) magnification) or strong (3). The fraction of PSMA positive cells was assessed as $<5 \%$ or $>5 \%$. In the case of heterogeneous staining, the predominant pattern was recorded. For further analysis, labeling indices were defined. A weak (1) or moderate (2) staining intensity in $<5 \%$ of the neovasculature and a weak (1) staining intensity in $>5 \%$ of the neovasculature was allocated to the "low expression" group (PSMA labelling index $=1$ ), whereas a moderate (2) staining intensity in $>5 \%$ of the neovasculature and a strong (3) staining intensity in $<$ or $>5 \%$ of the neovasculature were assigned to the "strong expression" group (PSMA labelling index $=2$ ). This scoring system has been previously established in mesenchymal tumors [34].

\section{Statistics}

SPSS 21 software (IBM, Armonk, NY, USA) was used. Fisher's exact was used if appropriate. All tests were two-sided with a $95 \%$ confidence interval.

\section{ACKNOWLEDGMENTS}

We thank Inka Buchroth (Münster) for technical assistance and Hans-Ulrich Schildhaus (Göttingen, Germany) for consultation.

\section{CONFLICTS OF INTERESTS}

The authors declare that there is no conflict of interests associated with this manuscript.

\section{GRANT SUPPORT}

The Deutsche Forschungsgemeinschaft (DFG) provided financial support to KS (grant STE 2467/1-1). This work was supported by the fund "Innovative Medical Research" of the University of Münster Medical School to MT and SH (grant HU121421).

\section{REFERENCES}

1. Carter RE, Feldman AR, Coyle JT. Prostate-specific membrane antigen is a hydrolase with substrate and pharmacologic characteristics of a neuropeptidase. Proc Natl Acad Sci U S A. 1996; 93: 749-53.

2. Pinto JT, Suffoletto BP, Berzin TM, Qiao CH, Lin S, Tong WP, May F, Mukherjee B, Heston WD. Prostate-specific membrane antigen: a novel folate hydrolase in human prostatic carcinoma cells. Clin Cancer Res. 1996; 2: 144551.

3. Troyer JK, Beckett ML, Wright GL, Jr. Detection and characterization of the prostate-specific membrane antigen (PSMA) in tissue extracts and body fluids. Int J Cancer. 1995; 62: 552-8.

4. Silver DA, Pellicer I, Fair WR, Heston WD, Cordon-Cardo C. Prostate-specific membrane antigen expression in normal and malignant human tissues. Clin Cancer Res. 1997; 3: 815 .

5. Ross JS, Sheehan CE, Fisher HA, Kaufman RP, Jr., Kaur P, Gray K, Webb I, Gray GS, Mosher R, Kallakury BV. Correlation of primary tumor prostate-specific membrane antigen expression with disease recurrence in prostate cancer. Clin Cancer Res. 2003; 9: 6357-62.

6. Ahmadzadehfar H, Rahbar K, Kurpig S, Bogemann M, Claesener M, Eppard E, Gartner F, Rogenhofer S, Schafers M, Essler M. Early side effects and first results of radioligand therapy with (177)Lu-DKFZ-617 PSMA of castrate-resistant metastatic prostate cancer: a two-centre study. EJNMMI Res. 2015; 5: 114.

7. Kratochwil C, Giesel FL, Stefanova M, Benesova M, Bronzel M, Afshar-Oromieh A, Mier W, Eder M, Kopka K, Haberkorn U. PSMA-targeted radionuclide therapy of metastatic castration-resistant prostate cancer with $\mathrm{Lu}-177$ labeled PSMA-617. J Nucl Med. 2016. 
8. Ghosh A, Heston WD. Tumor target prostate specific membrane antigen (PSMA) and its regulation in prostate cancer. J Cell Biochem. 2004; 91: 528-39.

9. Bostwick DG, Pacelli A, Blute M, Roche P, Murphy GP. Prostate specific membrane antigen expression in prostatic intraepithelial neoplasia and adenocarcinoma: a study of 184 cases. Cancer. 1998; 82: 2256-61.

10. Evangelista L, Briganti A, Fanti S, Joniau S, Reske S, Schiavina R, Stief C, Thalmann GN, Picchio M. New Clinical Indications for F/C-choline, New Tracers for Positron Emission Tomography and a Promising Hybrid Device for Prostate Cancer Staging: A Systematic Review of the Literature. Eur Urol. 2016.

11. Foss CA, Mease RC, Cho SY, Kim HJ, Pomper MG. GCPII imaging and cancer. Curr Med Chem. 2012; 19: 1346-59.

12. Bander NH, Trabulsi EJ, Kostakoglu L, Yao D, Vallabhajosula S, Smith-Jones P, Joyce MA, Milowsky M, Nanus DM, Goldsmith SJ. Targeting metastatic prostate cancer with radiolabeled monoclonal antibody J591 to the extracellular domain of prostate specific membrane antigen. J Urol. 2003; 170: 1717-21.

13. Chang SS, Reuter VE, Heston WD, Bander NH, Grauer LS, Gaudin PB. Five different anti-prostate-specific membrane antigen (PSMA) antibodies confirm PSMA expression in tumor-associated neovasculature. Cancer Res. 1999; 59: 3192-8.

14. Wang HL, Wang SS, Song WH, Pan Y, Yu HP, Si TG, Liu Y, Cui XN, Guo Z. Expression of prostate-specific membrane antigen in lung cancer cells and tumor neovasculature endothelial cells and its clinical significance. PLoS One. 2015; 10: e0125924.

15. Wernicke AG, Edgar MA, Lavi E, Liu H, Salerno P, Bander NH, Gutin PH. Prostate-specific membrane antigen as a potential novel vascular target for treatment of glioblastoma multiforme. Arch Pathol Lab Med. 2011; 135: 1486-9.

16. Godeiro KD, Frota AC, Antecka E, Odashiro AN, Maloney S, Fernandes B, Burnier MN, Jr. Prostate-specific membrane antigen is undetectable in choroidal neovascular membrane. J Carcinog. 2006; 5: 21.

17. Chang SS, O'Keefe DS, Bacich DJ, Reuter VE, Heston WD, Gaudin PB. Prostate-specific membrane antigen is produced in tumor-associated neovasculature. Clin Cancer Res. 1999; 5: 2674-81.

18. Wang W, Tavora F, Sharma R, Eisenberger M, Netto GJ. PSMA expression in Schwannoma: a potential clinical mimicker of metastatic prostate carcinoma. Urol Oncol. 2009; 27: 525-8.

19. Zeng C, Ke ZF, Yang Z, Wang Z, Yang SC, Luo CQ, Wang LT. Prostate-specific membrane antigen: a new potential prognostic marker of osteosarcoma. Med Oncol. 2012; 29: 2234-9.

20. Conway RE, Petrovic N, Li Z, Heston W, Wu D, Shapiro LH. Prostate-specific membrane antigen regulates angiogenesis by modulating integrin signal transduction.
Mol Cell Biol. 2006; 26: 5310-24.

21. Fletcher CD. The evolving classification of soft tissue tumours - an update based on the new 2013 WHO classification. Histopathology. 2014; 64: 2-11.

22. Group ESESNW. Soft tissue and visceral sarcomas: ESMO Clinical Practice Guidelines for diagnosis, treatment and follow-up. Ann Oncol. 2014; 25 Suppl 3: iii102-12.

23. Van Glabbeke M, van Oosterom AT, Oosterhuis JW, Mouridsen H, Crowther D, Somers R, Verweij J, Santoro A, Buesa J, Tursz T. Prognostic factors for the outcome of chemotherapy in advanced soft tissue sarcoma: an analysis of 2,185 patients treated with anthracycline-containing firstline regimens--a European Organization for Research and Treatment of Cancer Soft Tissue and Bone Sarcoma Group Study. J Clin Oncol. 1999; 17: 150-7.

24. Sleijfer S, Ouali M, van Glabbeke M, Krarup-Hansen A, Rodenhuis S, Le Cesne A, Hogendoorn PC, Verweij J, Blay JY. Prognostic and predictive factors for outcome to first-line ifosfamide-containing chemotherapy for adult patients with advanced soft tissue sarcomas: an exploratory, retrospective analysis on large series from the European Organization for Research and Treatment of Cancer-Soft Tissue and Bone Sarcoma Group (EORTC-STBSG). Eur J Cancer. 2010; 46: 72-83.

25. Patrikidou A, Domont J, Cioffi A, Le Cesne A. Treating soft tissue sarcomas with adjuvant chemotherapy. Curr Treat Options Oncol. 2011; 12: 21-31.

26. Milowsky MI, Nanus DM, Kostakoglu L, Sheehan CE, Vallabhajosula S, Goldsmith SJ, Ross JS, Bander NH. Vascular targeted therapy with anti-prostate-specific membrane antigen monoclonal antibody J591 in advanced solid tumors. J Clin Oncol. 2007; 25: 540-7.

27. Von Hoff DD, Mita MM, Ramanathan RK, Weiss GJ, Mita AC, LoRusso PM, Burris HA, 3rd, Hart LL, Low SC, Parsons DM, Zale SE, Summa JM, Youssoufian H, et al. Phase I Study of PSMA-Targeted Docetaxel-Containing Nanoparticle BIND-014 in Patients with Advanced Solid Tumors. Clin Cancer Res. 2016.

28. Rahbar K, Weckesser M, Huss S, Semjonow A, Breyholz HJ, Schrader AJ, Schafers M, Bogemann M. Correlation of Intraprostatic Tumor Extent with 68Ga-PSMA Distribution in Patients with Prostate Cancer. J Nucl Med. 2016; 57: 563-7.

29. Afshar-Oromieh A, Hetzheim H, Kratochwil C, Benesova M, Eder M, Neels OC, Eisenhut M, Kubler W, HollandLetz T, Giesel FL, Mier W, Kopka K, Haberkorn U. The Theranostic PSMA Ligand PSMA-617 in the Diagnosis of Prostate Cancer by PET/CT: Biodistribution in Humans, Radiation Dosimetry, and First Evaluation of Tumor Lesions. J Nucl Med. 2015; 56: 1697-705.

30. Ahmadzadehfar H, Eppard E, Kurpig S, Fimmers R, Yordanova A, Schlenkhoff CD, Gartner F, Rogenhofer S, Essler M. Therapeutic response and side effects of repeated radioligand therapy with 177Lu-PSMA-DKFZ-617 of castrate-resistant metastatic prostate cancer. Oncotarget. 
2016; 7: 12477-88. doi: 10.18632/oncotarget.7245.

31. Fina L, Molgaard HV, Robertson D, Bradley NJ, Monaghan P, Delia D, Sutherland DR, Baker MA, Greaves MF. Expression of the CD34 gene in vascular endothelial cells. Blood. 1990; 75: 2417-26.

32. Schlingemann RO, Rietveld FJ, de Waal RM, Bradley NJ, Skene AI, Davies AJ, Greaves MF, Denekamp J, Ruiter DJ. Leukocyte antigen CD34 is expressed by a subset of cultured endothelial cells and on endothelial abluminal microprocesses in the tumor stroma. Lab Invest. 1990; 62: 690-6.
33. Siemerink MJ, Klaassen I, Vogels IM, Griffioen AW, Van Noorden CJ, Schlingemann RO. CD34 marks angiogenic tip cells in human vascular endothelial cell cultures. Angiogenesis. 2012; 15: 151-63.

34. Schildhaus HU, Riegel R, Hartmann W, Steiner S, Wardelmann E, Merkelbach-Bruse S, Tanaka S, Sonobe H, Schule R, Buettner R, Kirfel J. Lysine-specific demethylase 1 is highly expressed in solitary fibrous tumors, synovial sarcomas, rhabdomyosarcomas, desmoplastic small round cell tumors, and malignant peripheral nerve sheath tumors. Hum Pathol. 2011; 42: 1667-75. 